\title{
The Fractality of the Hydrodynamic Modes of Diffusion
}

\author{
P. Gaspard and I. Claus \\ Center for Nonlinear Phenomena and Complex Systems \\ Université Libre de Bruxelles, Code Postal 231,Campus Plaine, B-1050 Brussels, Belgium \\ T. Gilbert \\ Department of Chemical Physics, \\ The Weizmann Institute of Science, Rehovot 76100, Israel \\ J. R. Dorfman \\ Department of Physics and Institute for Physical Science and Technology, \\ University of Maryland, College Park MD, 20742, USA
}

(October 29, 2018)

\begin{abstract}
Transport by normal diffusion can be decomposed into the so-called hydrodynamic modes which relax exponentially toward the equilibrium state. In chaotic systems with two degrees of freedom, the fine scale structure of these hydrodynamic modes is singular and fractal. We characterize them by their Hausdorff dimension which is given in terms of Ruelle's topological pressure. For longwavelength modes, we derive a striking relation between the Hausdorff dimension, the diffusion coefficient, and the positive Lyapunov exponent of the system. This relation is tested numerically on two chaotic systems exhibiting diffusion, both periodic Lorentz gases, one with hard repulsive forces, the other with attractive, Yukawa forces. The agreement of the data with the theory is excellent.
\end{abstract}

PACS numbers: 05.45.Df; 05.45.Ac; 05.60.-k; 05.70.Ln

Recent progress has revealed very interesting relationships between non-equilibrium statistical mechanics and and fractal structures that appear in the phase space of non-equilibrium, chaotic systems. Theoretical and numerical work has shown that systems of interacting particles have a high sensitivity to initial conditions as characterized by positive Lyapunov exponents [1, 2, 3, 3,4 , 5, 67. As a consequence, the motion of the particles composing the system is typically chaotic. Under stationary or time-dependent non-equilibrium conditions, this dynamical chaos generates fractal structures in phase space [7, 8,9, 10, 11, 12, 13, 14, 15, 16, 17]. In this context, Gilbert et al. 18 have very recently shown for a multibaker model of diffusion that the entropy production expected from non-equilibrium thermodynamics is a consequence of the fractal character of the hydrodynamic modes. Moreover, the same authors have shown that the diffusion coefficient is related to the fractal dimension of these modes in a family of multibaker models $[19$. The important question remains whether such results can be extended to more realistic systems.

The purpose of this Letter is to describe the fractality of the hydrodynamic modes of diffusion for general chaotic systems with two degrees of freedom, including Hamiltonian ones. The present approach requires neither an escape of particles out of the system as in the escaperate formalism, nor a non-Hamiltonian thermostat as in the thermostatted-system approach. Both formalisms lead to connections between transport coefficients and chaotic properties, but in a restrictive framework, which is not the case here. We obtain a formula which gives the Hausdorff dimension of the hydrodynamic modes in terms of: (a) the dispersion relation of diffusion, and (b) the Ruelle topological pressure which characterizes the chaotic properties of the dynamics. This new formula allows us to prove that, for general chaotic systems with two degrees of freedom, the diffusion coefficient is given in terms of the Hausdorff dimension of the modes and the positive Lyapunov exponent, as conjectured in Ref. [19. The results are numerically tested for two examples of Hamiltonian Lorentz gases.

The relaxation rate of a hydrodynamic mode of wavenumber $\mathbf{k}$ is given by the decay rate of the Van Hove intermediate incoherent scattering function [20] as

$$
s_{\mathbf{k}}=\lim _{t \rightarrow \infty} \frac{1}{t} \ln \left\langle\exp \left[i \mathbf{k} \cdot\left(\mathbf{r}_{t}-\mathbf{r}_{0}\right)\right]\right\rangle,
$$

where $\langle\cdot\rangle$ denotes an average over an ensemble of initial conditions and $\mathbf{r}$ denotes the position of the tracer particle. We assume that the system is mixing so that this average weakly converges toward its equilibrium value. The diffusion coefficient $\mathcal{D}$ can be obtained by expanding the relaxation rate (1) in powers of the wavenumber as

$$
s_{\mathbf{k}}=-\mathcal{D} \mathbf{k}^{2}+\mathcal{O}\left(\mathbf{k}^{4}\right),
$$

which is known as the dispersion relation of diffusion [21]. In deterministic chaotic systems, the hydrodynamic modes of diffusion turn out to be given by a singular distribution without density function [22]. For singular distributions, a cumulative function can nevertheless be defined by

$$
F_{\mathbf{k}}(\theta) \equiv \lim _{t \rightarrow \infty} \frac{\int_{0}^{\theta} d \theta^{\prime} \exp \left\{i \mathbf{k} \cdot\left[\mathbf{r}_{t}\left(\theta^{\prime}\right)-\mathbf{r}_{0}\left(\theta^{\prime}\right)\right]\right\}}{\int_{0}^{2 \pi} d \theta^{\prime} \exp \left\{i \mathbf{k} \cdot\left[\mathbf{r}_{t}\left(\theta^{\prime}\right)-\mathbf{r}_{0}\left(\theta^{\prime}\right)\right]\right\}},
$$


where the initial conditions form a one-dimensional line parameterized by the angle $\theta \in[0,2 \pi[$. We suppose that the system has two degrees of freedom so that each trajectory has one unstable, one stable, and two neutral directions in the four-dimensional phase space. The onedimensional line of initial conditions is assumed to be transverse to the unstable directions. The denominator in Eq. (3), allows us to define a normalized cumulative function such that $F_{\mathbf{k}}(0)=0$ and $F_{\mathbf{k}}(2 \pi)=1$. The cumulative function forms a curve $\left(\operatorname{Re} F_{\mathbf{k}}, \operatorname{Im} F_{\mathbf{k}}\right)$ in the complex plane $\mathbb{C}$. This curve is invariant under the dynamics and it gives the fine structure of the hydrodynamic mode of relaxation along the given one-dimensional line in phase space.

If we assume that the system is chaotic with the Axiom-A properties, we can use a result by Sinai, Bowen and Ruelle that averages can be performed in terms of a subset of unstable trajectories covering the phase space within a certain resolution [23,24]. As the time $t$ and the resolution increase the number of these trajectories also increases so as to fill densely the phase space in the limit $t \rightarrow \infty$. The probability weight given to each of these trajectories is inversely proportional to their stretching factor $\Lambda_{t}^{(j)}$ which characterizes its dynamical instability. We notice that the Lyapunov exponent of the trajectory is given by $\lambda^{(j)}=\lim _{t \rightarrow \infty}(1 / t) \ln \left|\Lambda_{t}^{(j)}\right|$. Under these circumstances, the average of a quantity $A$ can be expressed in the limit $t \rightarrow \infty$ as $\langle A\rangle \sim \sum_{j}\left|\Lambda_{t}^{(j)}\right|^{-1} A^{(j)}$, where $A^{(j)}$ is the quantity evaluated for the $j^{\text {th }}$ trajectory. Accordingly, Eq. (II) can be transformed into the condition

$\sum_{j}\left|\Lambda_{t}^{(j)}\right|^{-1} \exp \left(-s_{\mathbf{k}} t\right) \exp \left\{i \mathbf{k} \cdot\left[\mathbf{r}_{t}^{(j)}-\mathbf{r}_{0}^{(j)}\right]\right\} \sim_{t \rightarrow \infty} 1$.

Indeed, Eq. (1) is obtained after taking the logarithm of Eq. (何), dividing by $t$, and taking the limit $t \rightarrow \infty$.

Now, let us suppose that the sum in Eq. (位) is restricted to the trajectories issued from initial conditions in the interval $[0, \theta]$. In this case, we obtain at time $t$ a polygonal approximation of the cumulative function Eq. (3) because, the integral $\int_{0}^{\theta}$ is an average over trajectories with initial conditions in $[0, \theta]$ and the denominator is proportional to the factor $\exp \left(s_{\mathbf{k}} t\right)$. In this respect, we notice that Eq. (4) with a complete sum is equivalent to the condition $F_{\mathbf{k}}(2 \pi)=1$. Therefore, we can conclude that, at time $t$, the curve $\left(\operatorname{Re} F_{\mathbf{k}}, \operatorname{Im} F_{\mathbf{k}}\right) \subset \mathbb{C}$ is approximated by a polygon of sides given by the small complex vectors

$$
\Delta F^{(j)}=\left|\Lambda_{t}^{(j)}\right|^{-1} \exp \left(-s_{\mathbf{k}} t\right) \exp \left\{i \mathbf{k} \cdot\left[\mathbf{r}_{t}^{(j)}-\mathbf{r}_{0}^{(j)}\right]\right\}
$$

as in the construction of fractal curves of von Koch's type [25]. Each side has the length

$$
\varepsilon_{j}=\left|\Delta F^{(j)}\right|=\left|\Lambda_{t}^{(j)}\right|^{-1} \exp \left(-\operatorname{Re} s_{\mathbf{k}} t\right),
$$

so that the polygon can be covered by balls of diameter $\varepsilon_{j}$. In the limit $t \rightarrow \infty$, this polygon converges to a fractal curve characterized by a Hausdorff dimension given by $\sum_{j} \varepsilon_{j}^{D_{\mathrm{H}}} \sim 1$. Accordingly, the Hausdorff dimension of the hydrodynamical mode should satisfy the condition

$$
\sum_{j}\left|\Lambda_{t}^{(j)}\right|^{-D_{\mathrm{H}}} \exp \left(-D_{\mathrm{H}} \operatorname{Re} s_{\mathbf{k}} t\right) \sim_{t \rightarrow \infty} 1 .
$$

On the other hand, Ruelle's topological pressure is defined in dynamical systems theory by $[13,15,26$

$$
P(\beta) \equiv \lim _{t \rightarrow \infty} \frac{1}{t} \ln \left\langle\left|\Lambda_{t}\right|^{1-\beta}\right\rangle .
$$

Since there is no escape of trajectories in our case, we have that $P(1)=0$. The mean positive Lyapunov exponent of the system is given by $\lambda=-d P /\left.d \beta\right|_{\beta=1}$. Equation (8) can be transformed in the same way as Eq. (1) is transformed into Eq. (4), so that

$$
\sum_{j}\left|\Lambda_{t}^{(j)}\right|^{-\beta} \exp [-P(\beta) t] \sim_{t \rightarrow \infty} 1 .
$$

Comparing with Eq. (7), we infer that $\beta=D_{\mathrm{H}}$ whereupon

$$
P\left(D_{\mathrm{H}}\right)=D_{\mathrm{H}} \operatorname{Re} s_{\mathbf{k}},
$$

which is the new and central formula of this Letter. This formula generalizes Bowen's formula $P\left(D_{\mathrm{H}}\right)=0$ for the Hausdorff dimension of a fractal invariant set of trajectories [27]. In the present case, we are instead dealing with a complex invariant curve associated with a relaxation process of rate $s_{\mathrm{k}}$, which explains the presence of a new term in the right-hand side of Eq. (10). The root of Eq. (10) gives the Hausdorff dimension $D_{\mathrm{H}}(\mathbf{k})$ of the hydrodynamic mode of wavenumber $\mathbf{k}$.

If the wavenumber $\mathbf{k}$ vanishes, the relaxation rate (1) also vanishes, $s_{\mathbf{k}=0}=0$, and Eq. (3) shows that the cumulative function becomes $F_{\mathbf{k}=0}(\theta)=\theta /(2 \pi)$, which forms a straight line in the complex plane. In this equilibrium limit, Eq. (10) reduces to $P\left(D_{\mathrm{H}}\right)=0$ so that we get the dimension $D_{\mathrm{H}}=1$, as it should be for a straight line.

For a non-vanishing but small wavenumber, the Hausdorff dimension is expected to deviate from unity. Inserting $D_{\mathrm{H}}=1+\delta$ and the dispersion relation (2) in Eq. (10), we can expand both sides in powers of the wavenumber by using the aforementioned properties of the topological pressure. This straightforward calculation shows that the Hausdorff dimension of the hydrodynamic mode is given by

$$
D_{\mathrm{H}}(\mathbf{k})=1+\frac{\mathcal{D}}{\lambda} \mathbf{k}^{2}+\mathcal{O}\left(\mathbf{k}^{4}\right)
$$


as previously obtained for the multibaker models 19 . Accordingly, we have proved the conjecture of Ref. [19], that for general chaotic systems with two degrees of freedom, the diffusion coefficient can be expressed in terms of the positive Lyapunov exponent $\lambda$ and the Hausdorff dimension of the hydrodynamic modes as

$$
\mathcal{D}=\lambda \lim _{\mathbf{k} \rightarrow 0} \frac{D_{\mathrm{H}}(\mathbf{k})-1}{\mathbf{k}^{2}} .
$$

We numerically tested the relation (12) for periodic Lorentz gases, either with repulsive or with attractive scatterers. For the case of repulsive scatterers, we considered the periodic Lorentz gas where a point particle undergoes elastic collisions on hard disks forming a triangular lattice. This billiard is known to be fully chaotic 28]. If the horizon seen by the moving particle is finite the diffusion coefficient is positive and finite [28, 29], and the higher-order coefficients such as the super-Burnett coefficients are finite [30]. We take the radius of the disks to be unity, and the distance between the centers of the disks equal to $d=2.3$, so that the finite-horizon condition $2<d<4 / \sqrt{3}$ is satisfied. Numerical computation shows that the diffusion coefficient is $\mathcal{D}=0.25 \pm 0.01$ and the positive Lyapunov exponent $\lambda=1.76 \pm 0.01$. For this system, the curves $\left(\operatorname{Re} F_{\mathbf{k}}, \operatorname{Im} F_{\mathbf{k}}\right)$ of the hydrodynamic modes of wavenumbers $k_{x}=0.0,0.5$, and 0.9 and $k_{y}=0$ are depicted in Fig. 1. The initial conditions of the point particle are taken as the one-dimensional line given in phase space by the positions $\mathbf{r}_{0}(\theta)=(\cos \theta, \sin \theta)$ and the velocities $\mathbf{v}_{0}(\theta)=(\cos \theta, \sin \theta)$. The Hausdorff dimension of the fractal curves of Fig. 11 has been computed by a box-counting algorithm for different values of the wavenumber. These values are plotted versus $k_{x}^{2}$ in Fig. 2 (filled circles) together with a solid line of slope $\mathcal{D} / \lambda=0.14$. The very nice agreement shows that the dimension behaves as expected from Eq. (11), which provides numerical evidence of the validity of Eq. (12).

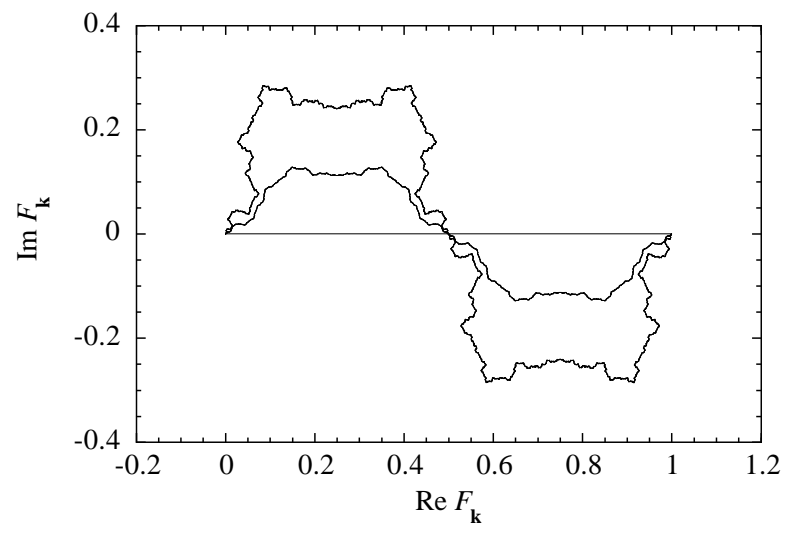

FIG. 1. Periodic Lorentz gas where a point particle of unit mass and velocity undergoes elastic collisions on hard disks of unit radius forming a triangular lattice with interdisk distance $d=2.3$ : Curves of the cumulative functions of the hydrodynamic modes of wavenumber $k_{x}=0.0,0.5$, and 0.9 with $k_{y}=0$. Note that the fractality increases with the wavenumber. The curves are constructed by averaging over $10^{6}$ points.

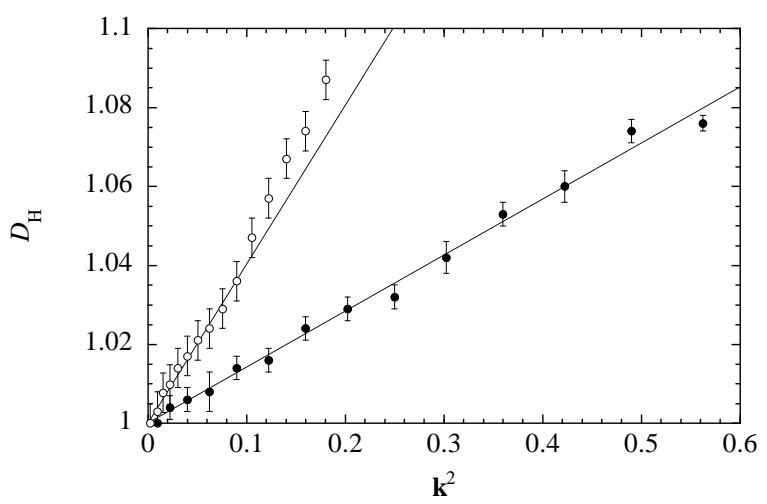

FIG. 2. Hausdorff dimension $D_{\mathrm{H}}$ of the hydrodynamic modes versus $\mathbf{k}^{2}=k_{x}^{2}\left(k_{y}=0\right)$ for both periodic Lorentz gases with hard-disk scatterers (filled circles) and Coulomb scatterers (open circles). Both solid lines have slopes equal to $\mathcal{D} / \lambda$ for the respective diffusion coefficient $\mathcal{D}$ and Lyapunov exponent $\lambda$ of the Lorentz gases.

For the case of attractive scatterers, we have considered the periodic Lorentz gas where an electrically charged particle of mass $m$ moves in a square lattice of screened Coulomb potentials (also known as Yukawa potentials). The Hamiltonian of this system is

$$
H=\frac{p_{x}^{2}+p_{y}^{2}}{2 m}-\sum_{\mathbf{l}} \frac{\exp (-\alpha\|\mathbf{r}-\mathbf{l}\|)}{\|\mathbf{r}-\mathbf{l}\|},
$$

where $\mathbf{r}=(x, y), \mathbf{l}=\left(l_{x}, l_{y}\right)$ with $l_{x}, l_{y}=0, \pm 1, \pm 2, \pm 3, \ldots$ and $\|\cdot\|$ is the Euclidean distance. This system was proved by Knauf to be fully chaotic and diffusive if the energy of the moving particle is positive and large enough [31]. We have here taken the parameter values $m=1, \alpha=2$, and the energy $E=3$. We have numerically computed the diffusion coefficient $\mathcal{D}=2.5 \pm 0.1$ and the Lyapunov exponent $\lambda=6.2 \pm 0.1$. The curves $\left(\operatorname{Re} F_{\mathbf{k}}, \operatorname{Im} F_{\mathbf{k}}\right)$ of the hydrodynamic modes of wavenumbers $k_{x}=0.0,0.2$, and 0.4 and $k_{y}=0$ are depicted in Fig. 3. The initial conditions of the point particle are taken on a circle centered around the scatterer at $x=y=0$, with positions $\mathbf{r}_{0}(\theta)=0.25(\cos \theta, \sin \theta)$ and velocities $\mathbf{v}_{0}(\theta)=v_{0}(\cos \theta, \sin \theta)$ such that $E=3$. The Hausdorff dimension of these fractal curves are also plotted in Fig. 2 (open circles), now together with a solid line of slope $\mathcal{D} / \lambda=0.40$. Here again, we observe a good agreement between both the numerical data and the theoretical prediction of Eq. (11). In this case, the data points deviate from the solid line at large values of $k_{x}^{2}$. 
Such a behavior is due to the terms of order $\mathbf{k}^{4}$ and higher in Eq. (11). These terms turn out to be more important in the case of the Coulomb scatterers than in the case of the hard-disk scatterers. Nevertheless, the data points converge to the unit value tangentially to the solid line, as predicted by Eq. (11). Therefore, we have here also a good confirmation of Eq. (12) for the Hamiltonian system (13).

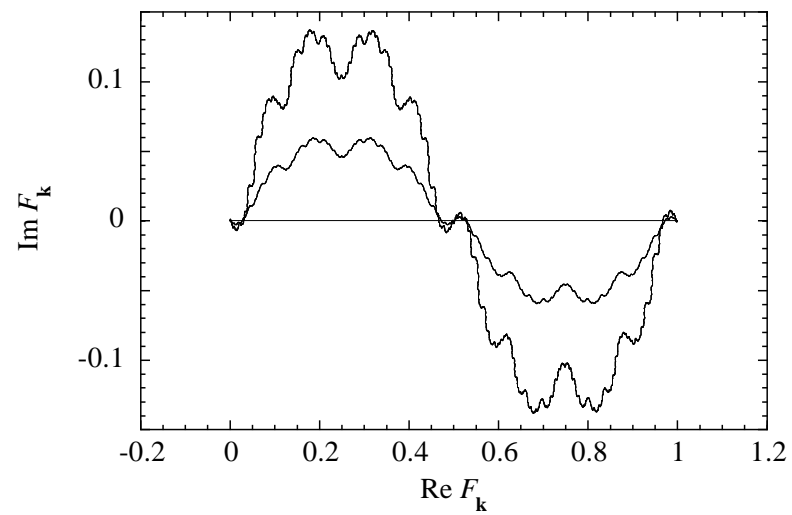

FIG. 3. Periodic Lorentz gas where a charged particle of unit mass and energy $E=3$ moves in a square lattice of screened Coulomb potentials with $\alpha=2$ : Curves of the cumulative functions of the hydrodynamic modes of wavenumber $k_{x}=0.0,0.2$, and 0.4 with $k_{y}=0$. Note that here also the fractality increases with the wavenumber. The curves are constructed by averaging over $10^{6}$ points.

In conclusion, we have derived the formula 10 for the Hausdorff dimension of the hydrodynamic modes of diffusion in chaotic systems with two degrees of freedom. This formula generalizes Bowen's formula and it allows us to establish in more generality a relation between the diffusion coefficient, the Lyapunov exponent, and the Hausdorff dimension of the hydrodynamic modes as conjectured in Ref. 19]. Our results exhibit new relations between transport coefficients and chaotic properties for systems relaxing to equilibrium, extending those previously obtained in the escape-rate formalism [7, 13, 14 and in the Gaussian thermostat method [8,9:10.11.17]. Therefore, they illustrate the great importance of such relationships for both Hamiltonian and non-Hamiltonian systems. Extensions of these and related results to fluid systems are now under study by the present authors.

We thank C. Dettmann, G. Nicolis, S. Tasaki, and Howard Weiss for discussions. JRD thanks the National Science Foundation for support under grant PHY 9820824. TG is a Feinberg Postdoctoral Fellow. IC and PG thank the FNRS Belgium and the IAP Program of the Belgian Federal OSTC for financial support.
[1] N. N. Krylov, Nature 153, 709 (1944); N. N. Krylov, Works on the Foundations of Statistical Mechanics (Princeton University Press, 1979); Ya. G. Sinai, ibid. p. 239.

[2] Ya. G. Sinai, Russian Math. Surveys 25, 137 (1970).

[3] R. Livi, A. Politi, and S. Ruffo, J. Phys. A: Math. Gen. 19, 2033 (1986).

[4] H. A. Posch and W. G. Hoover, Phys. Rev. A 38, 473 (1988); Ch. Dellago, H. A. Posch, and W. G. Hoover, Phys. Rev. E 53, 1485 (1996).

[5] H. van Beijeren and J. R. Dorfman, Phys. Rev. Lett. 74, 4412 (1995); 76, 3238 (1996); H. van Beijeren, J. R. Dorfman, H. A. Posch, and Ch. Dellago, Phys. Rev. E 56, 5272 (1997); R. van Zon, H. van Beijeren, and Ch. Dellago, Phys. Rev. Lett. 80, 2035 (1998).

[6] N. I. Chernov, J. Stat. Phys. 88, 1 (1997).

[7] P. Gaspard and G. Nicolis, Phys. Rev. Lett. 65, 1693 (1990).

[8] D. J. Evans, E. G. D. Cohen, and G. Morriss, Phys. Rev. A 42, 5990 (1990).

[9] D. J. Evans and G. Morriss, Statistical Mechanics of Nonequilibrium Liquids, (Academic Press, London, 1990).

[10] W. Hoover, Computational Statistical Mechanics, (Elsevier Science Publishers, Amsterdam, 1991).

[11] N. I. Chernov, G. L. Eyink, J. L. Lebowitz, and Ya. G. Sinai, Phys. Rev. Lett. 70, 2209 (1993); Commun. Math. Phys. 154, 569 (1993).

[12] S. Tasaki and P. Gaspard, J. Stat. Phys. 81, 935 (1995).

[13] P. Gaspard and F. Baras, Phys. Rev. E 51, 5332 (1995).

[14] J. R. Dorfman and P. Gaspard, Phys. Rev. E 51, 28 (1995); P. Gaspard and J. R. Dorfman, Phys. Rev. E 52, 3525 (1995).

[15] P. Gaspard, Chaos, Scattering, and Statistical Mechanics (Cambridge University Press, Cambridge, 1998).

[16] J. R. Dorfman, An Introduction to Chaos in Nonequilibrium Statistical Mechanics (Cambridge University Press, Cambridge UK, 1999).

[17] C. P. Dettmann, The Lorentz gas as a paradigm for nonequilibrium stationary states; and T. Tél and J. Vollmer, Entropy balance, multibaker maps, and the dynamics of the Lorentz gas, in: D. Szasz, Editor, Hard Ball Systems and Lorentz Gas, Encycl. Math. Sci. (Springer, Berlin, 2000).

[18] T. Gilbert, J. R. Dorfman, and P. Gaspard, Phys. Rev. Lett. 85, 1606 (2000).

[19] T. Gilbert, J. R. Dorfman, and P. Gaspard, Fractal dimensions of the hydrodynamic modes of diffusion, (preprint hlin.CD/0007008, server xxx.lanl.gov).

[20] L. Van Hove, Phys. Rev. 95, 249 (1954). (Dover, New York, 1980).

[21] H. van Beijeren, Rev. Mod. Phys. 54, 195 (1982).

[22] P. Gaspard, Phys. Rev. E 53, 4379 (1996).

[23] Ya. G. Sinai, Russian Math. Surveys 27, 21 (1972).

[24] R. Bowen and D. Ruelle, Invent. Math. 29, 181 (1975).

[25] H. von Koch, translated in: Classics on Fractals, G. A. Edgar, Ed. (Addison-Wesley, Reading MA, 1993); H. von Koch, Acta Mathematica 30, 145 (1906).

[26] D. Ruelle, Thermodynamic Formalism (Addison-Wesley, Reading MA, 1978).

[27] R. Bowen, Publ. Math. IHES 50, 11 (1976).

[28] L. A. Bunimovich and Ya. G. Sinai, Commun. Math. Phys. 78, 247, 479 (1980). 
[29] N. I. Chernov, J. Stat. Phys. 74, 11 (1994).

[30] N. I. Chernov and C. P. Dettmann, Physica A 279, 37 (2000); C. P. Dettmann, The Burnett expansion of the periodic Lorentz gas (preprint nlin.CD/0003038, server xxx.lanl.gov).

[31] A. Knauf, Commun. Math. Phys. 110, 89 (1987); Ann. Phys. (N. Y.) 191, 205 (1989). 